\title{
The Design of Continuous Wave Light Source on Detecting Aflatoxin
}

\author{
Xuyang Zhao ${ }^{2, \text { a }}$, Hao Yang ${ }^{1, \text { b, }}{ }^{*}$, and Cancan Huang ${ }^{2, \mathrm{c}}$ \\ ${ }^{1}$ College of Information Engineering China Jiliang University Hangzhou 310000, China \\ ${ }^{2}$ Yangtze Delta Region Institute of Tsinghua University Zhejiang Jiaxing 314000, China \\ azhaoxuyangmail@126.com, b, *16317367@qq.com, ‘493920925@qq.com
}

\begin{abstract}
The separation of elements is achieved by ultra-performance liquid chromatography system for Aflatoxins, the use of a closed-loop feedback control system is to produce a stable continuous wave light source, fixed wavelength of $360 \mathrm{~nm}$ illumination light source onto the separation of elements, resulting in the emission wavelength was $440 \mathrm{~nm}$ receiving photomultiplier tube, and then it gets high-frequency pulses. By single photon counting system pulse will be integrated for a fixed time, aflatoxin can be obtained G1, B1, G2, B2 content elements through the peak area calculation. Without derivatives quantitative determination of aflatoxin can be carried out quickly and efficiently. This design can effectively address the impact of the stability of the light source on the test results. It is useful for analysis LED spectral characteristics.
\end{abstract}

Keywords: closed loop feedback; stable light source; fixed wavelength; high frequency; single Photon Counting.

\section{基于连续波光源的黄曲霉毒素检测研究}

\section{赵许阳 ${ }^{2}$ ，杨昊 ${ }^{1}$, 黄灿灿 ${ }^{2}$}

1. 中国计量学院 信息工程学院 杭州 中国;

\section{2. 浙江清华长三角研究院 嘉兴 中国}

摘要: 采用超高效液相色谱系统对黄曲霉毒素进行元素分离, 使用闭环反馈控制系统设计连续 波光源产生稳定光源, 光源产生的 $360 \mathrm{~nm}$ 的固定波长照射到分离的元素上, 产生 $440 \mathrm{~nm}$ 的发射 波长被光电倍增管接收, 转化为高频脉冲。通过单光子计数系统对固定时间脉冲积分, 间接 获得黄曲霉毒素的G1、B1、G2、B2元素的含量. 无需衍生即可快速高效的进行黄曲霉毒素的定 量测定. 该设计能够有效的解决光源的稳定性对检测结果的影响, 适用于LED光谱特性的分析.

关键字: 闭环反馈; 稳定光源; 固定波长; 高频脉冲; 单光子计数

\section{1. 引言}

黄曲霉毒素是黄曲霉、寄生曲霉和模式曲霉等在合适的温度和湿度的环境下产生的真菌霉素, 对人畜有强烈的致病性、致癌性, 严重危害人体健康, 是危害最严重的真菌霉素, 在自然界 中广泛的存在 $[1,2]$. 黄曲霉毒素主要有 G1、B1、G2、B2 元素. 传统的黄曲霉毒素检测方法主 要有薄层层析法、超高效液相色谱法、免疫化学分析方法. 薄层层析法存在特异性不强, 容易 被其他荧光物质干扰, 前处理较为繁琐的缺点. 免疫化学分析方法只能测定黄曲霉毒素总量 [3]. 为了快速检测食品中的黄曲霉毒素的含量, 根据范德米特方程理论, 随着液相色谱系统 颗粒度的不断降低, 色谱分离度不断的提高, 因此采用超高效液相色谱法能够建立一种所需 样品量小、分离度高、快读准确的黄曲霉毒素定量分析方法 [4].

\section{2. 黄曲霉毒素检测原理}

超高效液相色谱法测定黄曲霉毒素的成分含量主要采用的单光子计数的方法, 采用单光子计 数方式具有集成度高、检测精度高、稳定度好等特点利用弱光照射下光子探测器输出电信号 
离散的特点, 把微弱的信号识别并提取出来, 在一定时间的积分范围内进行光子计数, 通过 曲线绘制和数据处理测定黄曲霉毒素中 G1、B1、G2、B2 元素的含量 [5].

连续波光源是化学发光分析测量系统主要的部分, 连续波光源发射出固定波长 $370 \mathrm{~nm}$ 的紫外 光, 照射在分离元素上将会激发出 $440 \mathrm{~nm}$ 的反射波, 反射波被光电倍增管的光阴极接受后将 会产生离散型的脉冲信号, 通过电脉冲信号, 就可以测到光子的个数, 将采集到的数据绘制 成曲线, 根据波峰的面积在上位机软件处理后就可以获取待测黄曲需溶液的浓度值 $[6,7]$.

\section{3. 连续波光源的设计以及分析}

黄曲霉毒素的检测需要在甲醇溶剂中进行测定, 在未加入黄曲霉毒素的甲醇溶剂中光源也会 激发出一定数目的光子数. 在检测时需要维持光源的稳定, 以保持基线检测时基线始终维持在 相对未定的范围内. 在实验时也可以通过基线间接的检测光源的稳定性 $[8,9,10]$.

3.1 连续波光源的硬件设计

在系统中需要相对简单并且精准的光源模块, 因此选择采用的放光二极管具有光强反馈和电 流反馈的双反馈闭环控制功能，可以实现长期自动监控调节，有效的保证了光源的稳定性. 根据实验所需的光源的特点, 由 LED 灯的光谱曲线图图 1 可知, 光源产生的波长需要在 $370 \mathrm{~nm}$.

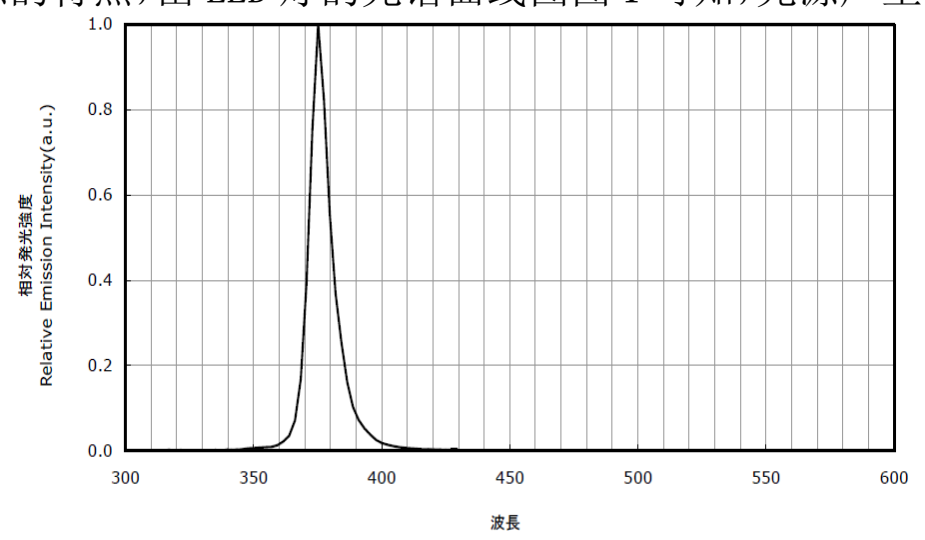

图 1 LED 灯的光谱曲线图

对于 LED 灯的光源的稳定性控制, 则主要是使用 ADN2830 控制器, 光源的反馈则主要是采用 光电池 S1087 作为反馈量大小的主要器件. ADN2830 控制器能够为连续波光源提供闭环的反 馈回路, 控制回路通过监视的 LED 的电流并实时的提供稳定的电流, 以维持恒定的光功率. 采用该控制器的优点是可以通过小规模改变外部电路设计, 就可以调整所需的光功率.

在光源反馈环节主要采用的由 OP2177 组成的 I/V 转换电路. 控制器提供电源点亮 LED, LED 产 生的光强被光电池接受转化为电流输出, 经过 I/V 转换电路转换为电压反馈到控制器, 控制 器将会根据所需的光强进行自动调节. 由于光电池产生的电流较小达到 $\mathrm{uA}$ 级, 因此在选择具 有精密、单位增益稳定、低噪声、低输入的偏置电流运算放大器. 光强反馈调节电路图如图 2.



图 2 光强反馈调节电路图 


\section{4. 硬件测试数据以及分析}

连续波光源的硬件设计的主要目标是获取长时间稳定的光源为黄曲霉毒素的元素检测提供合 适的光源, 光源的稳定性主要体现在直接的光源电路的电压、电流以及光功率的数据采集, 长时间的检测基线以及在实际的黄曲霉毒素检测中的曲线的平稳度中.

对光源电路的稳定性验证首先是通过采集光源电路的电压、电流以及光功率有限性数据. 表 1 是光源电路的电压、电流以及光功率数据.

表 1 光源电路的电压、电流以及光功率数据

\begin{tabular}{llll}
\hline 采集时间/ & 电流/ & 电压/ & 光功率/ \\
$\min$ & $\mathrm{mA}$ & $\mathrm{V}$ & $\mathrm{mW}$ \\
\hline 0 & 25.31 & 3.428 & 4.7 \\
30 & 25.32 & 3.425 & 4.8 \\
60 & 25.34 & 3.423 & 4.7 \\
120 & 25.32 & 3.423 & 4.7 \\
\hline
\end{tabular}

通过以上的数据可以较为直观的看出光源的工作电压以及工作电流, 根据 LED 的数据参考手 册可知 LED 灯工作在额定参数之内, 光功率式中保持稳定. 从以上数据可以保证 LED 灯能够长 期稳定有效工作.

本设计中连续波光源是在黄曲霉毒素检测中使用的, 通过在检测黄曲霉毒素含量的过程中的 上位机的显示, 也可以清晰的分辨出光源的稳定性. 图 3 是基线检测波形图.

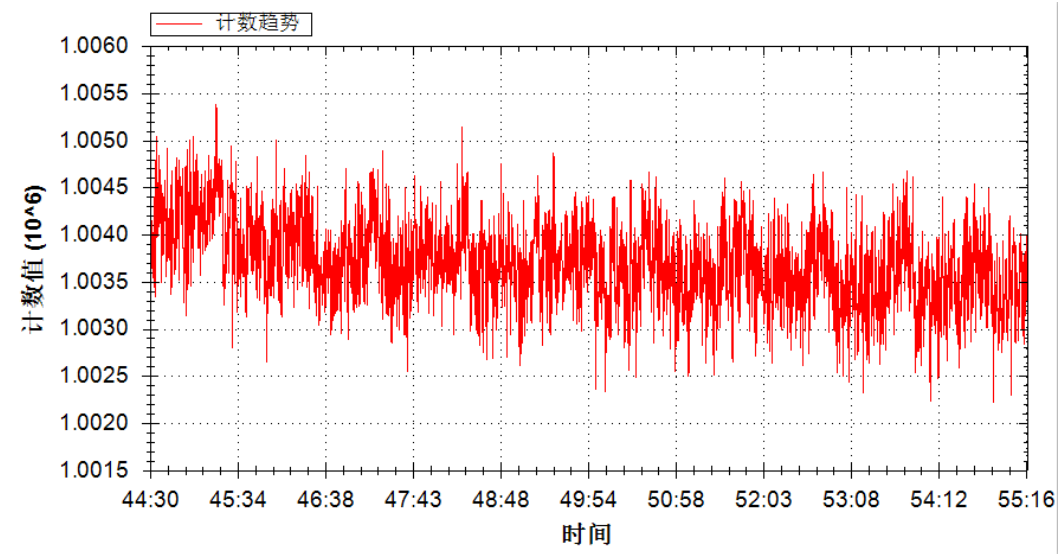

图 3 基线检测波形图

黄曲霉毒素 G1、B1、G2、B2 的显示曲线如图 4.

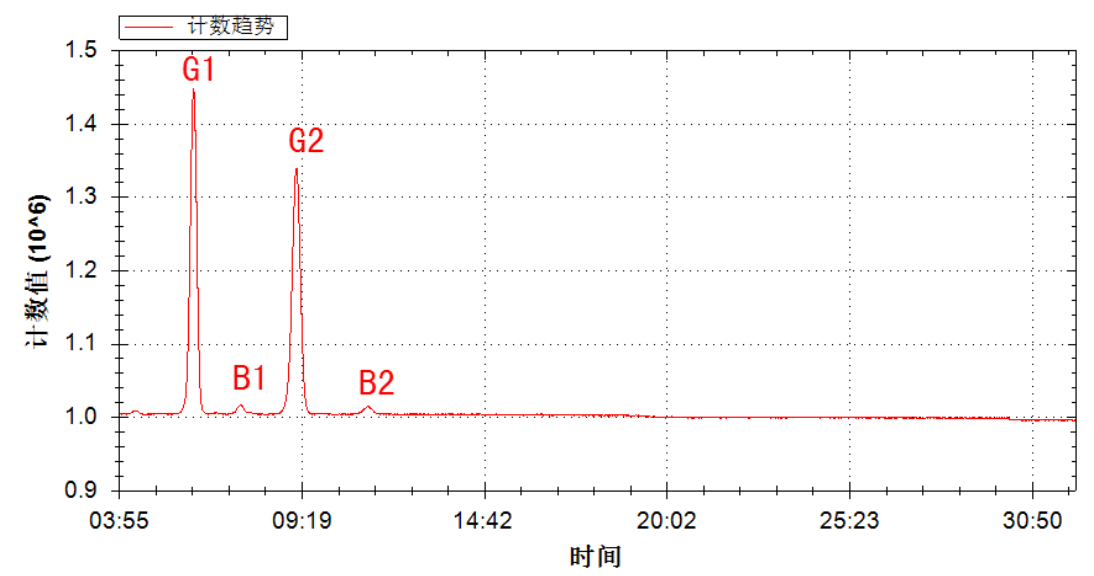

图4 黄曲霉毒素G1、B1、G2、B2的显示曲线 
图3是在黄曲霉毒素检测之前的系统稳定性测试曲线. 从基线的数据曲线可以看出跳动的范围 在 2000 左右, 在允许的误差范围内, 说明光源维持在相对稳定的状态. 经过基线检测之后注入 黄曲霉毒素出现G1、B1、G2、B2四个波峰, 能够清晰的分辨出各个元素的波形, 在之后的曲 线式中卫视在相对平稳的状态. 从以上的采集到的数据和测试获取的图片能够有效的说明连 续波光源的设计满足稳定性的要求，能够有效的对黄曲霉毒素进行元素分析.

\section{致谢}

浙江省科技计划项目 (2015C33009), 嘉兴市科技计划项目 (2015AY11008)

\section{References}

[1].Zain M E.Journal of Saudi Chemical Society [J].2011, 15(2):129-144.

[2].Lewis L,Onsongo M,Njapau H.Environ Health Perspect[J].2005,113(12):1763-1767.

[3].Chen Nan.Introduction the experience in the Aflaloxins testing [J]. Jiangxi Chemical Industry, 2009,4:9-11.

[4].Mather J， Rainville P D， Spooner N， Evans C A.Bioanalysis[J].2011,3(4):411-420

[5].Determination of Aflatoxinscontent in Food-Cleanup by Immunoaffinity Chromatography and Determination by High-performance Liquid Chromatography and Fluormeter[S].National Standards of the People,s Republic of China.GB/T 18979-2003.

[6].Li Weiyong, Chang Dading,Zeng Yanan.Single - Photon Counting System Research Based on CPLD[J]. Computer \& Digital Engineering, 2006, 38(6):84-88.

[7].Design and implementation of the single photon counter system [J]. Chinese Journal of Scientific Instrument, 2007,28(4): 28-30.

[8].Shi Yunfei,Jin Yi.Study on the Method for Determination of Aflatoxinin Peanut[J]. Chinese Journal of Health Laboratory Technology， 2012,22(11): 2584-2585.

[9].Peng Zhibing, Zhang Xuan,Jiang Jianyun.Analysis of Aflatoxin in Grain by Liquid-Liquid Extraction-High Performance Liquid Chromatography[J]. Grain Technology and Economy, 2013, 38(1):26-29.

[10].Chen Dongdong,Dai Hanxia,Peng Tao. Determination of six aflatoxins in animal liver by photochemical derivative-high performance liquid chromatography combining with immunoa ffinity column clean-up[J]. Journal of Food Safety and Quality, 2014, 5(3):796-800. 